\title{
Research on Guilin Smart Cultural Tourism Service Scenario based on Digital Twin Technology
}

\author{
Jingran Yang, Yuyuan Peng *, Hongjin Liang \\ School of Art and Design, Guilin University of Electronic Technology, Guilin, 541000, China \\ * pyy@guet.edu.cn
}

\begin{abstract}
In recent years, with the emergence of new technologies such as cloud computing, the Internet of Things, big data, and artificial intelligence, the country has deeply promoted the application of science and technology in the cultural and tourism industries, targeting smart cultural tourism, digital cultural industries, online and offline integrated development, and scenic spots. Multiple service scenarios such as smart supervision put forward new requirements. The purpose is to use digital twin technology to build a digital, online, and intelligent cultural tourism industry chain, optimize Guilin tourism service processes, improve service efficiency, and make full use of the city's smart cultural tourism development to promote the improvement of the city's image and promote Guilin The new form of global tourism forms the city's endogenous development capabilities, and provides theoretical and practical guidance for the construction of smart cultural tourism in other cities. By analyzing the current status of smart cultural tourism and application requirements, it discusses the design and construction of Guilin smart cultural tourism service scenarios based on digital twin technology, and how to strengthen the realization of future-oriented smart interactive scenarios in the innovation of service models.
\end{abstract}

Keywords: Digital Twin Technology; Guilin Smart Cultural Tourism; Intelligent Cultural Tourism Industry Chain.

\section{Introduction}

In 2018, it was mentioned in the "Explanation on the Institutional Reform Plan of the State Council": the establishment of the Ministry of Culture and Tourism. To promote the integrated development of cultural undertakings, cultural industries, and tourism, the plan proposes to integrate the responsibilities of the Ministry of Culture and the National Tourism Administration to form the Ministry of Culture and Tourism as a component of the State Council [1-2]. As a result, the contribution of the cultural and travel industry has become more and more important in the national economy, and the degree of policy attention has also increased. On September 11, 2020, Xin Guobin, Vice Minister of the Ministry of Industry and Information Technology, presided over the plenary meeting of the National Intelligent Manufacturing Standardization Coordination and Promotion Group, the General Group, and the Expert Advisory Group, and proposed to strengthen the forwardlooking layout of new technology application standards such as $5 \mathrm{G}$, artificial intelligence, and digital twins. With the application of 5G technology, the Internet of Things technology continues to mature, and the application of blockchain and artificial intelligence has gradually penetrated multiple industries and various fields. In particular, the application of 5G technology in the tourism industry marks the entry of China's tourism industry. "The Era of Wisdom Cultural Travel"[3]. In August 2013, Guilin was selected into the annual national smart city pilot list and officially launched the construction of a "smart city", using smart cultural tourism as a breakthrough point for smart cities to further improve the city's refined governance capabilities; at the same time, Guilin is tourism with unique regional and cultural characteristics Cultural cities need to be guided by governance upgrades and standardize the industrial form of the emerging tourism economy, provide cutting-edge smart cultural tourism services, strengthen smart planning and decision-making, and develop a global tourism pattern under the framework of digital twins [4].

The technology of digital twins drives the transformation of the tourism industry into a modern service and forms a new economic thrust through the release of the value of data assets. In the new era of smart operation in the cultural and tourism industry, the digital economy is integrated with the 
real economy to achieve a new dual-cycle development pattern; from cultural experience to online services, content innovation, information transmission, business integration, data assets, cross-border integration, and application of smart interaction Scenarios guide smart space design and accelerate the arrival of the popular smart tourism wave [5-6].

In short, digital twins have practical guiding significance for the construction of smart cultural tourism from theory to practice. Through the construction of "digital twins" smart cultural tourism theoretical methods and service design strategies, it is helpful to solve the outstanding problems of information islands in the development and construction of the tourism industry. According to actual scenarios, better governance and standardization of the digitalization of the tourism industry, promote the construction of the city's image and the appreciation of its tourism value and promote the investment and integration of a large number of emerging enterprises, thereby driving the economic development of surrounding industries. After the epidemic, smart cultural tourism will receive more attention from the industry, and smart disaster prevention, smart prediction, smart diversion, virtual reality experience, risk hedging tools based on big data, etc., will become areas that need more development [7].

\section{New Opportunities for the Development of Gunlin's Smart Cultural Tourism Industry}

As one of the application scenarios derived from the digital twin city, smart cultural tourism has gradually matured with culture as the connotation, tourism as the carrier, and technology as the driving force, continuously improving users' cultural perception and travel experience. The cultural tourism industry has begun to enter the highly intelligent development stage of the Internet of Everything. Smart cultural tourism will be driven by data innovation and led by scenario innovation. Through allfactor digital transformation and total-factor productivity improvement, it will comprehensively promote smart cultural tourism based on new infrastructure. New industry system construction.

Although Guilin Smart Tourism has achieved a certain degree of tourism informatization since its construction, it has provided information services such as online information publishing, online ticket purchase, and electronic guided tours, and some scenic spots have conducted some preliminary analysis and application of tourist data. Intelligent management has been realized in terms of monitoring passenger flow and safety management of scenic spots. Its shortcomings and outstanding problems are: unclear understanding of smart cultural tourism, confusion between smart cultural tourism and tourism informatization; insufficient planning and design of smart cultural tourism, lack of integrity and system; the degree of intelligence is not high enough; The system connectivity between the platforms is insufficient, the degree of integration and application of global tourism data is not high, and the supplementary effect of market information is limited.

Innovating Guilin's smart cultural tourism service scenario requires the integration of all service resources of the Guilin tourism industry, for tourists, tourism service providers, local people, and relevant government agencies to cooperate in receiving services, providing services, participating in services, and managing services. Solve the problems related to the development of smart cultural tourism, promote the whole life cycle decision-making of the scenic area in planning, construction, management, and operation, and solve the major challenges and key issues in the work of its digital service process.

\section{Smart Cultural Tourism Ecological Structure Model}

\subsection{Theoretical Integration Foundation}

From the perspective of the concept and development of digital twins, the academic community has mainly carried out related research on digital twin modeling, cyber-physical integration, interaction and collaboration, and service applications [2]. Its applications are mainly concentrated in the product design and management operation and maintenance phases of physical entities, providing 
important theoretical and technical support for the real-time association and interactive integration between physical entities and virtual spaces required for the construction of smart cultural tourism.

\subsection{Structure Model Foundation}

Tao Fei (2018) and others built a digital twin five- dimensional structure model and application guidelines, and provide users with functions such as intelligent applications, precise management, and reliable operation and maintenance generated by digital twin applications in the most convenient form while giving users the most intuitive interaction. Based on digital twin technology, intelligent recommendation and itinerary planning using smart technologies such as big data, cloud computing, artificial intelligence, refined and precise management, focusing on the endogenous development of the city, and changing the ecological structure of the domestic cultural and tourism industry, which is shown in Fig 1.

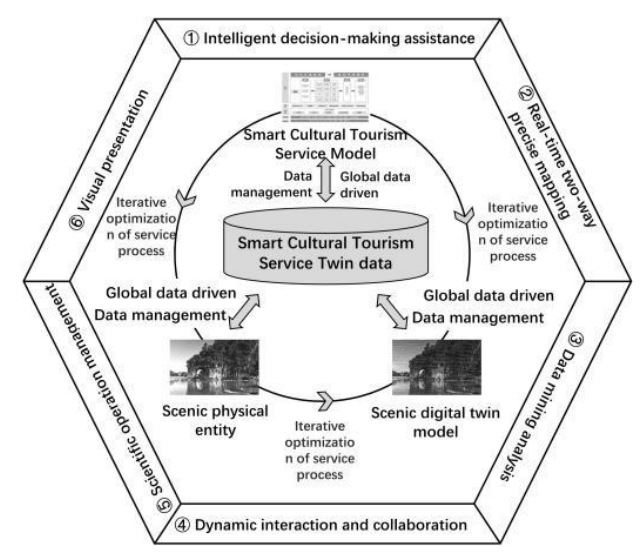

Fig 1. Structural model

\subsection{Ecological Model}

As shown in Fig.2, from a vertical perspective, it is divided into the application layer, service layer, data computing layer, network layer, and perception layer. The digital twin assists the government and tourism suppliers in the dynamic prediction and management of the entire industry chain and the entire life cycle; From a horizontal perspective, the final itinerary is based on structured semantic data to accurately model the actual scenic spot and the tourist experience process, forming a global intelligent environment interactive service perception system, through the management analysis system through the data engine to achieve integrated management, providing intelligence The construction of the analyzed ecological model; and the data integration and quantification of online and offline service supply platforms, to achieve multiple heterogeneities.

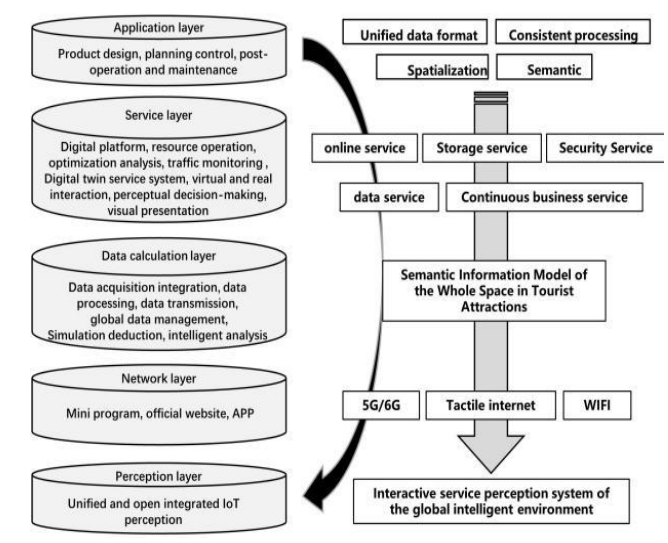

Fig 2. Smart cultural tourism ecological model 
In terms of service demand, only the provision of per- personalized tourism products and services can satisfy the free travel and individual travel form. Products and services are upgraded according to the characteristics of different groups of people. The provision of cultural content and the development of projects can also be tailored to the needs of the people. Provide differentiated products and services, and optimize service nodes in a targeted manner combined with the user journey map. In terms of service design dimensions, using multiple models and twin data as the core, we continue to develop and extend methods to link technology, cultural creation, and new media. In terms of service application dimensions, how to provide services that meet the needs of different fields, different levels of users, and different business application needs based on multi-dimensional models and twin data, and realize the value-added efficiency and efficiency of the on-demand use of services.

\section{Application of Digital Twin Technology in Guilin Smart Cultural Tourism Service Scenario}

\subsection{Operating Mechanism}

At present, Guilin tourism has achieved "seven upgrades", and is actively promoting the in-depth integration of cultural tourism to ensure that it will be fully built into a world-class international tourist destination by 2025. The operating mechanism of Guilin's smart cultural tourism ecology proposed in this paper is to build a virtual scenic spot model (digital twin) through digital twin technology, which completely digitally maps the scenic spot in the information virtual space, to target the tourist management, ecological monitoring, and equipment in the scenic spot. The maintenance and the protection, inheritance, development, and utilization of cultural heritage are faced with various situations and emergencies in the process of generating an optimized treatment plan.

\subsection{Construction Path}

The base technology architecture of the digital twin is used to support the optimization and upgrade of all levels of smart cultural tourism, as shown in Fig. 2. described as follows:

(1) The infrastructure directly serves the construction of smart cultural tourism in Guilin by new technologies, promotes the digital transformation of scenic spot construction, and provides technical support and implementation means for the development of smart cultural tourism and the formation of digital twins.

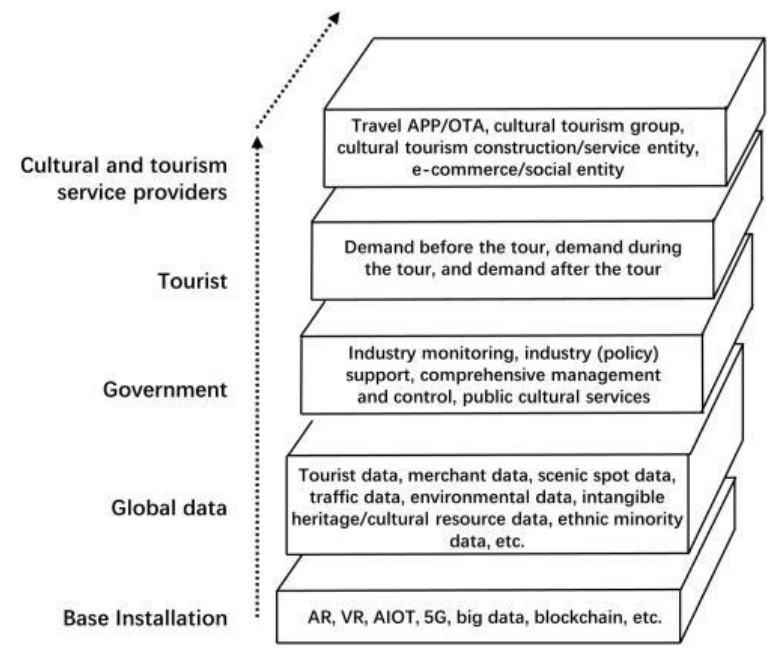

Fig 3. Guilin smart cultural tourism construction path based on digital twins

(2) The global data layer collects global tourism data in real-time, studies and analyzes the preferences and needs of tourists, provides advice and support for government decision-making, and provides support for the development of cultural experience service products. 
(3) At the government level, use data to improve the level of management, service, and decisionmaking, implement comprehensive management and control throughout the entire process, effectively integrate all aspects of Guilin's data resources, optimize the current Guilin tourism market order, and promote the construction of public services in the scenic area.

(4) For cultural and tourism service providers, use digital twin technology to build an open and innovative think tank platform, turn cultural scenes, and transform local traditional fine culture into experienceable, interactive, and entertaining tourism products.

Under the operation of the entire smart cultural and tourism ecological structure, from food, lodging, travel, shopping, and entertainment to business, education, learning, and leisure, smart cultural and tourism services run through each link and the whole process before, during, and after the tour, providing tourists with free Sewing, personalized, and experiential services.

\subsection{Innovation of Service Experience Model}

With the rapid development of the Internet, traditional sightseeing tourism can no longer meet the increasing high-level needs of tourists. Tourists pursuing a personalized experience journey should strengthen the innovation of service experience models and use digital twin technology to promote Guilin's wisdom. The specific measures for the development of cultural tourism are as follows:

(1) Provide personalized services centered on tourists. Today, new technologies such as the Internet of Things, big data, speech recognition, and image recognition have been applied to the cultural and tourism industry. The city is also full of various sensors and cameras; scenic spots and urban infrastructure, public service facilities, The operation status of traffic, people flow, scenic resources, etc., as well as the safe operation and control and deployment of municipal resources including police, medical, fire protection and other security, can use the terminal to extract, integrate and manage these big data information to control the physical level city of. Reasonable use of big data information can help tourists personally plan travel routes, order travel products, find service facilities, etc., optimize and upgrade scenic services, improve service processes, and solve users' worries.

(2) Enriching the tourism industry, integrating online and of- fline development, giving tourists a sense of participation and enhancing their satisfaction. With the help of virtualization technology, it can display the environment and related information, provide tourism products to create a more immersive tourism experience, generate new environments and visualization effects, enhance the scientific and technological interaction experience of the physical space, and more effectively activate the cultural tourism boutique Protect and promote.

(3) Construct a digital twin based on global tourism data and city models. Establish a service scene for smart cultural tourism, promote integrated development with culture, medical care, education, sports, business exhibitions, etc., to construct a smarter and more complete full-process service model for tourists.

\section{Conclusion}

In the process of digital, online, and intelligent transformation of the tourism industry, the integration of culture and tourism has been actively explored. At present, there are relatively little researches on smart cultural tourism in China, and there is a lack of architecture-level technological integration innovation solutions. Digital twins provide a guarantee from technology to theory to practice for the realization of smart cultural tourism models. There are few theoretical and applied types of research in this area, professional talents are scarce, and there are many shortcomings in the promotion and practice of new technologies. It will take some time to develop and test before largescale implementation. The realization of this new scenario of smart cultural tourism will promote the technological integration, business integration, and data integration of a new generation of information technology and urban construction, and promote the development and construction of software and hardware facilities and urban design of smart cultural tourism in space and place to user 
journey planning and construction. The integrated layout of the project explores the implementation of the smart cultural tourism service design model; it provides the tourism industry with an integrated solution from resources and services to the terminal for tourists and users, and will surely focus on security prevention and control, multi-party monitoring, real-time data, Wisdom experience, smart management, cultural interaction, and digital + IP marketing generate new value. Digitalization and intelligent management of online platforms are inevitable trends in the future development of the cultural travel industry. In the future, AI and VR/AR will further promote the improvement of service experience and the optimization and upgrading of tourism products and industries.

\section{References}

[1] China Unicom and Tencent, "China smart cultural tourism 5G application white paper," 2019.

[2] J. Shen, H. Xiang, and K. Jia, "Digital twin system and its construction based on the internet of things model of the electronics industry," Electric Power Information and Communication Technology, vol. 17, no. 03, pp. 26-31, 2019.

[3] F. Tao and M. Zhang, "Digital twin shop-floor: a new shop-floor paradigm towards smart manufacturing," IEEE Access, vol. 5, pp. 20 418-20 427, 2017.

[4] T. Fei, L. Weiran, L. Jianhua, and L. Xiaojun, "Digital twin and its application exploration," Computer Integrated Manufacturing System, vol. 24, no. 1, pp. 4-21, 2018.

[5] F. Tao, H. Zhang, Q. Qi, M. Zhang, W. Liu, J. Cheng, X. Ma, L. Zhang, and R. Xue, "Ten questions about digital twins: Analysis and thinking," Computer Integrated Manufacturing System, vol. 26, no. 1, pp. 521, 2020.

[6] China News, "The construction of Guilin international tourist resort in Guangxi has achieved "seven upgrades"," https://www.chinanews.com/ cj/2020/12-25/9371200.HTML, Accessed January 11, 2021.

[7] R. Song and X. Sun, "Laiwu "first-line five villages" ancient village tourism service design based on user experience," Art and Design (Theory), vol. 2, no. 4, pp. 108-112, 2019. 